\title{
Nonlinear Microwave Simulation Techniques
}

\author{
Almudena Suárez \\ Department of Communications Engineering, University of Cantabria, Santander, 39005, Spain
}

\begin{abstract}
The design of high performance circuits with short manufacturing cycles and low cost demands reliable analysis tools, capable to accurately predict the circuit behaviour prior to manufacturing. In the case of nonlinear circuits, the user must be aware of the possible coexistence of different steady-state solutions for the same element values and the fact that steadystate methods, such as harmonic balance, may converge to unstable solutions that will not be observed experimentally. In this contribution, the main numerical iterative methods for nonlinear analysis, including time-domain integrations, shooting, harmonic balance and envelope transient, are briefly presented and compared. The steady-state methods must be complemented with a stability steady-state analysis to verify the physical existence of the solution. This stability analysis can also be combined with the use of auxiliary generators to simulate the circuit self-oscillation and predict qualitative changes in the solution under the continuous variation of a parameter. The methods will be applied to timely circuit examples that are demanding from the nonlinear analysis point of view.

Index Terms - Time-domain integration, harmonic balance, envelope transient, stability.
\end{abstract}

\section{INTRODUCTION}

The growing demand for high-performance radiofrequency circuits and systems requires the use of accurate and efficient simulation tools. The simulation has added difficulties in the case of nonlinear circuits, which are governed by nonlinear differential equations [1-2]. In nonlinear dynamical systems there is a possible coexistence of steady-state solutions, which may be either stable (or robust under small perturbations) or unstable. Instability is often associated to the presence of unexpected self-sustained oscillations, only possible in nonlinear non-conservative systems [2]. In addition, two or more stable solutions may coexist for the same values of the circuit elements and parameters, the observation of one or another depending on the initial conditions. Different methods exist for the simulation of nonlinear circuits [3-12]. The analytical methods, such as the describing function or Volterra series [3-4], are well suited for circuit design, since they give insight into the nonlinear behavior and its dependence on the circuit parameters. However, when the goal is to obtain an accurate solution in terms of waveforms and spectral content, numerical iterative methods are generally preferred [4-12]. Time-domain integration provides the entire evolution of the circuit solution from the initial value to the steady state $[6,13]$. Provided that the integration step and the algorithm are properly selected, the steady-state solution obtained will be stable or physical. Fast time-domain methods, such as shooting [6] and finite-difference algorithms [14], perform the time-domain analysis of the steady state only, avoiding the transient through the use of an additional constraint. In timedomain methods, the modelling of linear distributed elements with loss and dispersion requires the calculation of impulse responses and convolution products [15-17]. In contrast, the harmonic balance (HB) method uses frequency-domain representations for the linear elements, lumped or distributed, maintaining the natural time domain descriptions for the nonlinear devices [9-12]. The circuit variables are represented by means of a Fourier series, with one or more fundamental frequencies, so only the steady state can be simulated. The method is unable to predict the transient reaction to perturbations and thus it is insensitive to the stability properties of the solution obtained [18-19]. The HB system of nonlinear algebraic equations is solved through an error minimization method, such that only the spectral components at the frequencies of the input generators are initialized by default. The resulting default solution may be unstable and never observed in practice. Therefore, the application of a complementary stability analysis is essential. A very practical method is the one based on pole-zero identification [20-21], relying on the fact that all the closed-loop transfer functions that can be defined in a linearized system share the same denominator and therefore exhibit the same poles. This method can be combined with the use of auxiliary generators (AGs) to simulate the possible circuit self-oscillation and predict qualitative changes in the solution under the continuous variation of a parameter [18-19]. This contribution presents several examples of timely circuits which are demanding from a simulation point of view, including pulse injection-locked oscillators [22-23], rotary travelling wave oscillators (RTWO) [24-25] and unstable power amplifiers.

\section{TIME-DOMAIN METHODS}

Time-domain integration relies on the formulation of the circuit as a set of differential algebraic equations (DAE) through the modified nodal approach (MNA) [26]:

$$
\bar{i}(\bar{v}(t))+\frac{d \bar{q}}{d t}(\bar{v}(t))+\int_{-\infty}^{t}[h(t-\tau)] \bar{v}(\tau) d \tau+\bar{g}(t)=0
$$

where $\bar{v} \in \mathrm{R}^{\mathrm{P}}$ is the vector containing the node voltages and branch currents, $\bar{q} \in \mathrm{R}^{\mathrm{P}}$ is the vector of charges and fluxes, $\bar{i} \in \mathrm{R}^{\mathrm{P}}$ are the sums of currents (that enter each node) and branch voltages, $[h]$ contains the impulsive responses of the distributed elements, and $\bar{g}(t) \in \mathrm{R}^{\mathrm{P}}$ are the input generators. Several algorithms [13] exist for the numerical integration of (1), depending on the way how $d \bar{q} / d t$ is approximated, in terms of the time samples. In implicit algorithms, $\bar{q}(\bar{v}(n+1))$ is a function of itself, as in 
$d \bar{q} / d t=\left(\bar{q}\left(\bar{v}\left(t_{n+1}\right)\right)-q\left(\bar{v}\left(t_{n}\right)\right)\right) /\left(t_{n+1}-t_{n}\right), \quad$ corresponding to Backward Euler's approximation. Replacing this expression into the MNA and discretizing the convolution operation, one obtains:

$$
\begin{aligned}
& \bar{e}\left(t_{n+1}\right) \equiv \frac{\bar{q}\left(\bar{v}\left(t_{n+1}\right)\right)-\bar{q}\left(\bar{v}\left(t_{n}\right)\right.}{t_{n+1}-t_{n}}+\bar{i}\left(\bar{v}\left(t_{n+1}\right)\right)+ \\
& +\sum_{i=1}^{n}\left[h\left(t_{n+1}-t_{i}\right)\right] \bar{v}\left(t_{i}\right) \Delta t_{i}+\bar{g}\left(t_{n+1}\right)=0
\end{aligned}
$$

The error function $\bar{e}\left(t_{n+1}\right)$ has to be minimized at each time step, which is usually done thorough a Newton-Raphson procedure. The trapezoidal approximation estimates the derivative $d \bar{q} / d t$ using the average of its value at $t_{n}$ and $t_{n+1}$. There are also more complex multi-step algorithms, which require two or more past points. A given algorithm has $M$ order when the solution $\bar{q}(\bar{v}(t))$ and its $M$ first time derivatives are continuous at the limits of the interval $\left[t_{n}, t_{n+1}\right]$. Provided that the integration step keeps relatively small, it will be possible to use a larger time step with a higher order algorithm. For a same continuous-time system, each algorithm gives rise to a different discrete-time equation. The stable solution of the continuous-time system may become an unstable solution of a particular discrete-time system. Problems are most common in systems having time constants of different order of magnitude, such as relaxation oscillators. Time domain integration has been applied for the analysis of a pulsed-injection locked oscillator (Fig. 1).

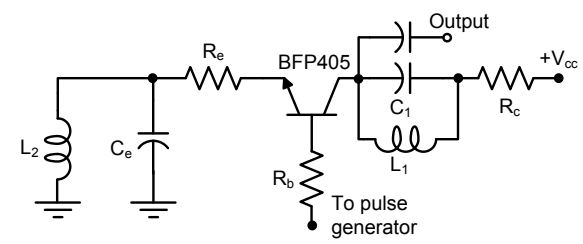

Fig. 1. Oscillator at $5.7 \mathrm{GHz}$ with an input pulse at $100 \mathrm{MHz}$ [23].

The objective is to reduce the phase noise of the higher frequency oscillator [22-23], operating at $5.7 \mathrm{GHz}$, through the synchronization to a pulsed signal of a much lower frequency, in the order of $100 \mathrm{MHz}$ (Fig. 2). The high ratio between the two frequencies prevents the use of harmonic balance, which would require a high number of harmonic terms. Furthermore, the frequency intervals with synchronized behaviour are delimited by instability phenomena and their prediction with $\mathrm{HB}$ would be demanding. The input signal switches the oscillation on and off, so the steady-state solution consists of a series of oscillation bursts. Fig. 2(a) and Fig. 2(b) show the simulated and measured synchronized solution for three different values of the pulse frequency. Fig. 2(c) and Fig. 2(d) show the oscillator waveform for two different values of the pulse frequency, with undesired frequency divisions by 2 [in (c)] and quasi-periodicity [in (d)]. Time domain integration can be combined with a Poincaré map technique $[13,23]$ to analyze the qualitative variations of the steady-state solution versus the pulse frequency $f_{p}$. At each $f_{p}$, the solution is examined in a time interval $\left(T_{\text {start }}, T_{\text {end }}\right)$, such that the circuit behaves in steady-state regime in this interval. The map is obtained by detecting all the relative maxima of the circuit solution in the analysis interval. If, in steady state, all the oscillation bursts are equal, they will all have the same maximum and only one point will be obtained. If the period of the steady-state solution is $n$-times the period of the input signal $T_{p}, n$ different maxima will be detected. If the oscillation bursts are not periodic, the burst maxima will have many different values and a distribution of points will be obtained. Fig. 3 shows the map resulting from the application of this technique.

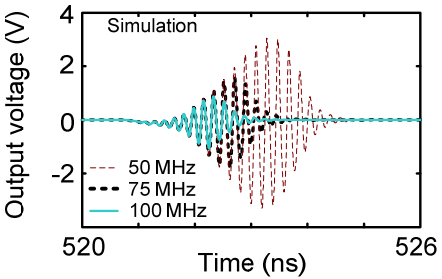

(a)

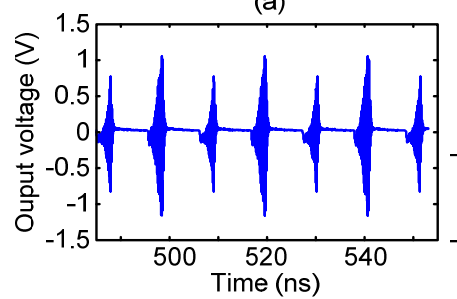

(c)

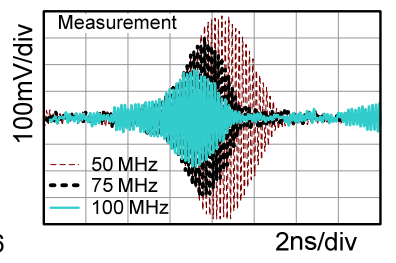

(b)

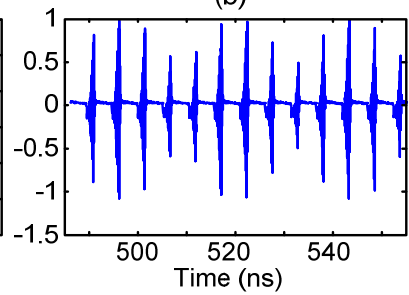

(d)
Fig. 2. Oscillator in Fig. 1. (a) Simulation in synchronized operation. (b) Measurement. (c) Divided by two regime. (d) Quasiperiodic solution.

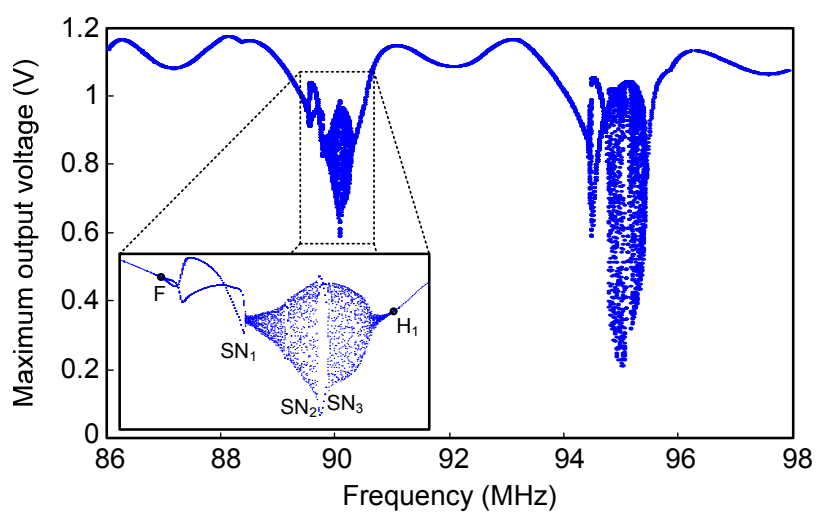

Fig. 3. Map of oscillation burst maxima versus the pulse frequency. Inset shows qualitative changes of the solution stability

The shooting methods [8,11-13] provide, through an optimization technique, a vector of initial conditions $\bar{v}_{o}$, from which the circuit behaves in steady-state regime. In the case of periodic solutions $\bar{v}(t)$, the constraint is $\bar{v}(0)-\bar{v}(T)=0$, with $T$ being the solution period. Let $\bar{v}(t)$ be expressed as: $\bar{v}(t)=\bar{v}\left(t_{o}\right)+\bar{\phi}\left(\bar{v}\left(t_{o}\right), 0, t\right)[6]$. Then, the shooting equation system is $\bar{\phi}\left(\bar{v}_{o}, 0, T\right)=0$, which contains $P$ equations in $P$ unknowns. To solve this system one must take into account that $\bar{v}\left(t_{n+1}\right)$ is an implicit function of $\bar{v}\left(t_{n}\right)$. A Newton- 
Raphson algorithm is used to solve $\bar{\phi}\left(\bar{v}_{o}, 0, T\right)=0$ in terms of $\bar{v}_{o}$. This requires the calculation of the sensitivity matrix $[J \phi] \equiv \partial \bar{\phi}\left(\bar{v}_{o}, 0, T\right) / \partial \bar{v}_{o}$. Thus, the circuit solved through a two-level Newton-Raphson algorithm. The outer level corresponds to the shooting equation system. The inner level corresponds to the time-domain integration through the interval $(0, T)$. Due to the limited length of this interval, the solution obtained could be unstable.

\section{HARMONIC BALANCE}

The HB technique [6,11-12] uses frequency-domain descriptions for the linear elements, while keeping the original time-domain descriptions for the nonlinear devices. In the nodal HB, the Fourier-series expansions of the vectors $\bar{v}(t), \bar{q}(t), \bar{i}(t), \bar{g}(t)$ are introduced into (1). Taking into account the orthogonality of the Fourier basis, one obtains the following relationship between the Fourier coefficients:

$$
\bar{E}(\bar{V}) \equiv \bar{I}(\bar{V})+[j \omega] \bar{Q}(\bar{V})+[H(j \omega)] \bar{V}+\bar{G}=0
$$

where $[j \omega]=\left[\operatorname{diag}\left(j \omega_{-N}\right) \ldots\left(j \omega_{k}\right) \ldots \operatorname{diag}\left(j \omega_{N}\right)\right], \quad N$ is the number of harmonic terms, and $\bar{E}(\bar{V})$ is an error function. Calculation of $\bar{I}(\bar{V})$ and $\bar{Q}(\bar{V})$ requires inverse $\left(\mathrm{F}^{-1}\right)$ and direct $(\mathrm{F})$ Fourier transforms. In the case of quasi-periodic regimes, a different time variable is associated to each fundamental frequency, performing a sequential calculation of fast Fourier transforms in the different time variables. The error function in (3) is usually minimized through the NewtonRaphson algorithm. Calculation of the Jacobian matrix requires the derivative matrixes $\partial \bar{I} / \partial \bar{V}, \partial \bar{Q} / \partial \bar{V}$ obtained from the harmonic components of the time-domain functions $\partial \bar{i} / \partial \bar{v}$ and $\partial \bar{q} / \partial \bar{v}$. Since the vectors $\bar{i}$ and $\bar{q}$ include both linear and nonlinear functions, the Jacobian matrix will have many zero elements (sparse matrix) [27-29]. This will enable the straightforward application of sparse-matrix techniques for linear-system solution, such as preconditioned Krylov subspace methods [27-29].

When the solution contains a self-oscillation at $f_{o}$, the HB system decomposes into a forced subsystem and a homogeneous subsystem at frequencies containing multiples of $f_{o}$. The later admits a zero solution, so the circuit can always be resolved for a non-oscillatory regime, which will be unstable in most cases [18-19]. To simulate the oscillatory solution, an AG operating at $F_{A G}=f_{o}$, with an amplitude $A_{A G}$ can be introduced into the circuit. The two variables $F_{A G}$ and $A_{A G}$ must be calculated in order to fulfil non-perturbation condition $Y_{A G}\left(A_{A G}, F_{A G}\right)=0$, where $Y_{A G}$ is the ratio between the AG current and voltage. This condition is resolved with the pure harmonic-balance system as an inner tier. The use of AGs enables an efficient frequency domain analysis of complex oscillator configurations, such as the recently proposed rotary travelling wave oscillator [24]. It is based on the use of Möbiusring-like differential transmission line, with gain stages periodically distributed along the path [24-25], which allows obtaining multi-phase solutions with a quasi-square waveform. Fig. 4 shows an implementation using sections of nonlinear transmission line (NLTL) that reduce the number of gain stages needed for the quasi-square shaping. Phase values at consecutive nodes should be $\phi_{m}=m 2 \pi /(2 N)$, with $m=0 \ldots 2 N-1$. However, there is a possible coexistence of oscillation modes, due to the system symmetries [25]. Assuming identical amplitudes, the total admittance matrix exhibits repeated eigenvalues, associated with different phase distributions. For the analysis/design of this oscillator configuration, $n_{A G}$ auxiliary generators are introduced through the structure (Fig. 4), fulfilling the conditions $Y_{A G i}=0$, where $i$ $=1$ to $n_{A G}$, which are solved simultaneously. This avoids undesired HB solutions having any of the gain stages in a non oscillatory state and allows presetting the phase shifts corresponding to each possible oscillation mode. The simulated and measured waveforms are compared in Fig. 5(a) and Fig. 5(b). The coexisting in-phase mode [Fig. 5(c)] is unstable. The variation of the oscillation frequency of the two modes versus the drain voltage is shown in Fig. 5(d).

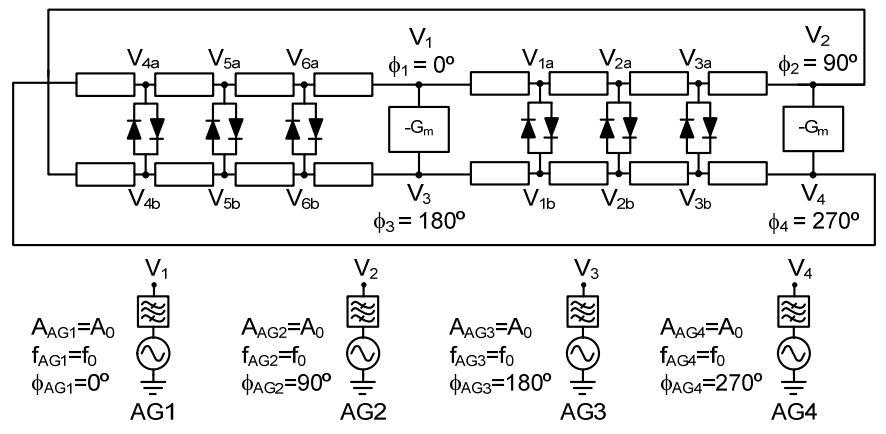

Fig. 4. NLTL-based rotary-travelling wave oscillator [25] at $\mathrm{f}_{\mathrm{o}}=700 \mathrm{MHz}$.

\section{STABILITY ANALYSIS}

In small-signal regime the circuit behaves linearly with respect to the input sources, so the stability properties only depend on the dc regime [18-19]. For the stability analysis, a small perturbation is considered, at the complex frequency $s$. Due to the small amplitude of the perturbation, the devices can be linearized about the dc solution. In the case of a largesignal periodic regime, the solution must be linearized about this periodic solution at the frequencies $j k \omega_{o}$, where $k=-N$ to $N$. Then the perturbation frequency $s$ gives rise to the mixing terms $j k \omega_{o}+s$. There are no generators at the perturbation frequencies, so one obtains a homogeneous system [11,18-19]: $\left[\frac{\partial \bar{I}\left(\bar{V}_{d c}\right)}{\partial \bar{V}}+[s] \frac{\partial \bar{Q}\left(\bar{V}_{d c}\right)}{\partial \bar{V}}+[H(s)]\right] \Delta \bar{V}=0$
$\left[\frac{\partial \bar{I}\left(\bar{V}_{p}\right)}{\partial \bar{V}}+\left[j k \omega_{o}+s\right] \frac{\partial \bar{Q}\left(\bar{V}_{p}\right)}{\partial \bar{V}}+\left[H\left(j k \omega_{o}+s\right)\right]\right] \Delta \bar{V}=0 L s$

where $S s$ and $L s$, respectively refer to a small- and largesignal stability analysis. To have $\Delta \bar{V} \neq 0$, the matrix affecting this vector must be singular or, equivalently, its determinant 
must be zero [11-12]. For the solution to be stable, all the determinant roots must have negative real part. In practice, the roots of the determinant can be obtained from pole-zero identification [20-21] of any closed-loop transfer function, obtained by connecting a small-signal source and linearizing the circuit about the steady-state solution.

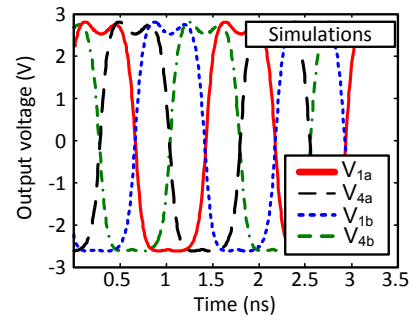

(a)

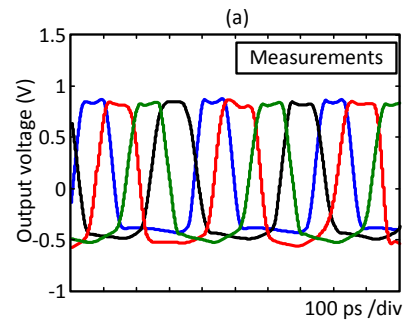

(b)

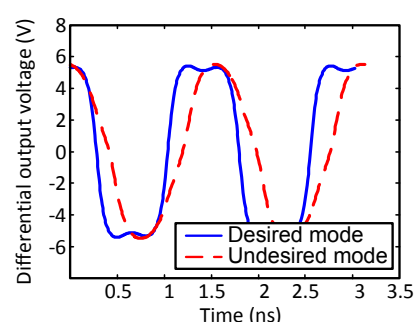

(c)

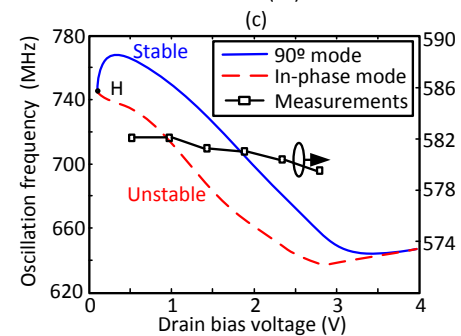

(d)
Fig. 5. Analysis of the RTWO. (a) Simulated waveforms of the stable multiphase mode. (b) Measured waveforms. (c) Multiphase (stable) and in-phase (unstable) mode. (d) Oscillation frequency vs. the drain bias voltage.

There can be a substantial difference between the default HB simulation and the physical solution of a given circuit. As an example, Fig. 6(a) presents the default HB solution of a power combining amplifier. The solution is periodic at the frequency of the input source for all the input power values. However, a stability analysis based on pole-zero identification [Fig. 6(b)] indicates that the solution is unstable in small signal, with a pair of complex-conjugate poles on the righthand side of the complex plane (RHS) at a frequency different from that delivered by the input source $\sigma \pm j\left(\omega_{i n} / 2 \pm \Delta \omega\right)$. At the input power $P_{\text {in }}=0.5 \mathrm{dBm}$, the pair of complexconjugate poles cross the imaginary axis to the left-hand side (LHS) and the default periodic solution becomes stable. When further increasing $P_{i n}$, the poles continue to evolve and at $P_{i n}=4 \mathrm{dBm}$, they merge and split into two pairs of poles at $\omega_{i n} / 2$. At $P_{\text {in }}=4.2 \mathrm{dBm}$, one of these two pairs of poles crosses the imaginary axis to the RHS and gives rise to a frequency division by 2 , so the periodic solution at $\omega_{\text {in }}$ becomes unstable again. Fig. 6(c) shows the stable quasiperiodic and frequency-divided steady-state solutions obtained with the aid of an AG. The output power has been represented versus $P_{i n}$ at each fundamental frequency. In the absence of input power, the circuit exhibits a free-running oscillation. This oscillation mixes with the frequency of the input drive up to the point $\mathrm{H}$ where the oscillation is extinguished. At F1, a subharmonic solution arises, which is extinguished at F2.

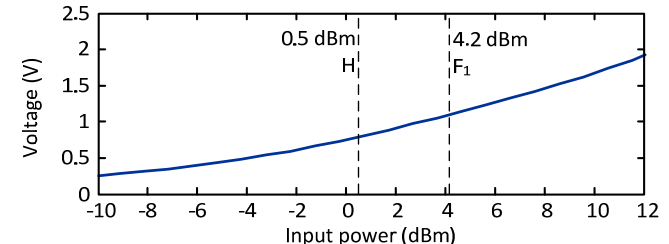

(a)

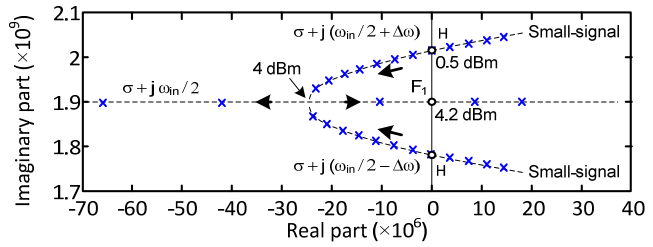

(b)

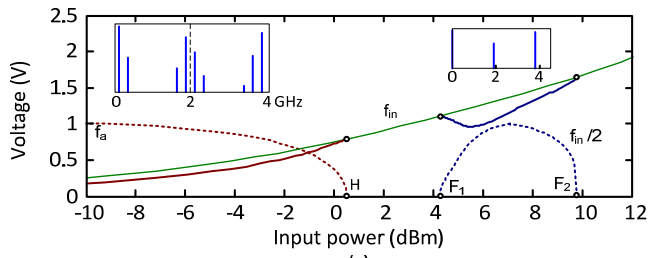

(c)

Fig. 6. Unstable power amplifier. (a) Default periodic solution in terms of drain voltage vs. $P_{i n}$. (b) Stability analysis vs. $P_{i n}$. (c) Stable quasi-periodic and divided-by-two solutions, obtained with an AG.

\section{ENVELOPE TRANSIENT}

Circuits containing modulated signals cannot be simulated with HB. However, in most cases, it is possible to consider two different time scales [30-31]. The faster time scale $t_{2}$ corresponds to the carrier and the slower time scale $t_{1}$ corresponds to the modulation. The circuit is generally periodic in the "faster" time $t_{2}$. Then, any state variable $a(t)$ can be expanded as: $a\left(t_{1}, t_{2}\right)=\sum_{k=-N}^{N} A_{k}\left(t_{1}\right) e^{j k \omega_{k} t_{2}}$, where $A_{k}\left(t_{1}\right)$ are slow varying envelopes. When these expressions are introduced in (1), the two time variables $t_{1}$ and $t_{2}$ will require two different derivative operators. For fixed $t_{1}$, the derivative with respect to $t_{2}$ will simply be obtained through the multiplication of the different harmonic terms by $j \omega_{k}$. This provides an algebraic system of differential equations in the harmonic terms $A_{k}\left(t_{1}\right)$. The time variable $t_{1}$ is discretized, replacing the time derivative with a particular expression in terms of the time samples, such as the backward Euler approximation. The integration time step $\Delta t_{1}$ is determined by the modulation rate, so it will be noticeably larger than the one required for full time-domain integration. Dropping the subindex in $t_{1}$, the envelope-transient equation takes the form:

$\bar{I}(\bar{V}(t))+[j \omega] \bar{Q}(\bar{V}(t))+\frac{d}{d t} \bar{Q}(\bar{V}(t))+[H] * \bar{V}(\tau) d \tau+\bar{G}(t)=0(5)$

The computation of the convolution products is less demanding than in standard time-domain integration, since the impulse responses can be narrowband about the harmonic frequencies $\omega_{k}$. The initial condition is obtained from an ordinary HB analysis at the initial time $t_{o}=0$. From this time value, the Newton-Raphson algorithm is applied to obtain the 
solution $\bar{V}(t)$ along the simulation interval. In the case of circuit containing self-oscillations, the oscillation frequency should be considered in the Fourier basis representation of the circuit variables [19,31]. However, the envelope sampling step may be too large to predict the frequency variations during the oscillation start-up. One way to cope with the problem is to perform an initial HB analysis (in the absence of modulation signals) with the aid of an AG [19]. The resulting solution is stored and used as initial value for the envelope-transient simulation. Alternatively, the AG may be connected to the circuit at the initial time only [19], using a time varying resistor $R(t)$ in a series connection, switching from zero to very high value at $t>t_{0}$.

In the absence of modulations, the amplifier analyzed in Fig. 7(a) exhibits a subharmonic instability for $P_{\text {in }}>4.2 \mathrm{dBm}$. An amplitude modulation of the input signal has been considered, such that this signal is above the "static" instability threshold for a fraction of the modulation period. For this dynamical analysis, the circuit variables will be represented in a Fourier basis at $f_{\text {in }} / 2$, with time-varying harmonic terms $A_{k}(t)$. The magnitude of the subharmonic component $A_{1}(t)$ for different modulation frequencies $f_{\bmod }$ is shown in Fig. 7(b), where the time axis has been normalized to the modulation period. The fraction of the period in which the subharmonic component is present decreases with $f_{\text {mod }}$. From certain $f_{\text {mod }}$, time above threshold is too short to enable the oscillation start-up [32].

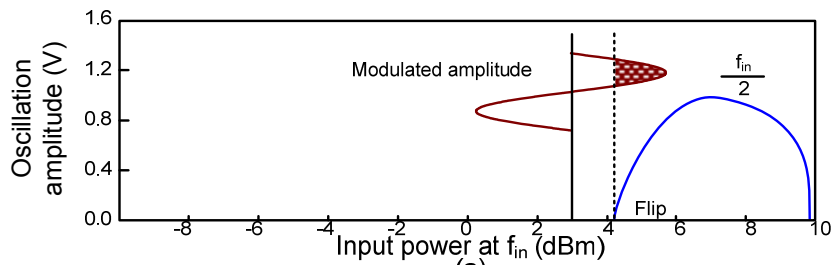

(a)

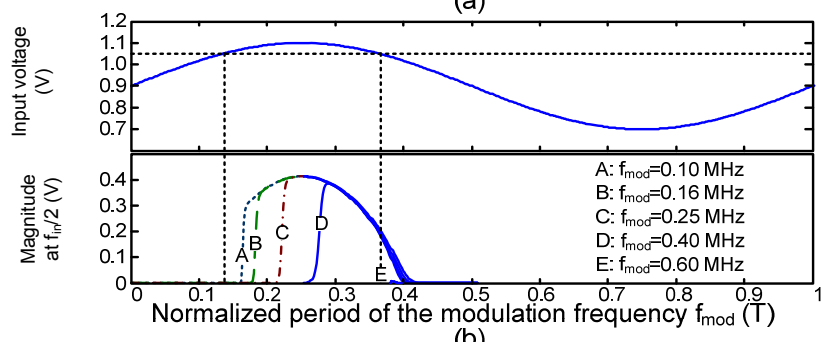

(b)

Fig. 7. Unstable amplifier with amplitude modulation. (a) Amplitude is above instability threshold for a fraction of modulation period. (b) Subharmonic component at $f_{\text {in }} / 2$ for different values of $f_{\text {mod. }}$.

Next example corresponds to a self-oscillating power amplifier (SOPA). In Class-D amplifiers, a high amplitude signal (switching signal) at the frequency $f_{o}$ makes the transistors switch between on- and off- stages and the output voltage has a rectangular waveform. In the case of a SOPA, the Class-D amplifier [32-34] is inserted into a feedback loop (Fig. 8), which should enable an oscillation at $f_{o}$, with no need for an extra source. The circuit has been analyzed with envelope transient, using an AG to initialize the oscillation at $f_{o}=750$ MHz. Fig. 9 presents the output power spectrum obtained with WCDMA-like signal centred at $200 \mathrm{MHz}$ [34]. It shows the dithering effect of the higher frequency oscillation. The impact of the input signal on this oscillation has been analyzed by means of two tones spaced $\Delta f \approx 2 \mathrm{MHz}$. High envelope excursions lead to an instantaneous reduction and even extinction of the oscillation amplitude, as shown in the time-domain analysis of Fig. 10(b), where denser traces are due to the self-oscillation. The envelope analysis in Fig. 10 (c) predicts the variation of the oscillation amplitude in an efficient and clear manner.

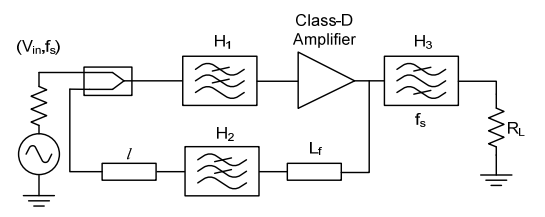

Fig. 8 Self-oscillating power amplifier with an oscillation at $f_{o}=750 \mathrm{MHz}$ and an input signal at $f_{i n}=200 \mathrm{MHz}$.

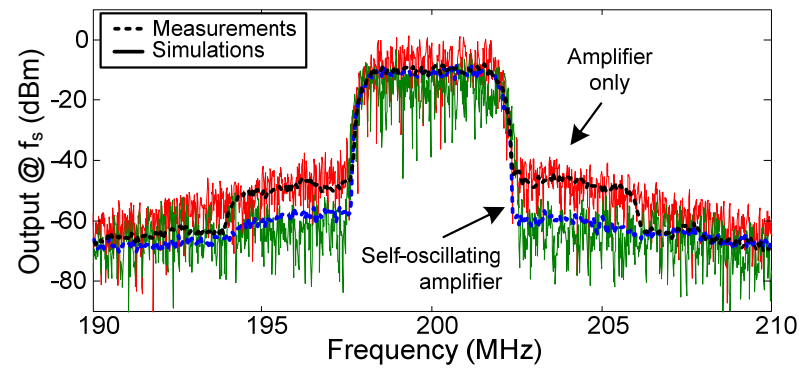

Fig. 9 Output-power spectrum of the SOPA obtained with a WCDMA signal [34]. Dashed line: measurements (averaged). Solid line: simulations.

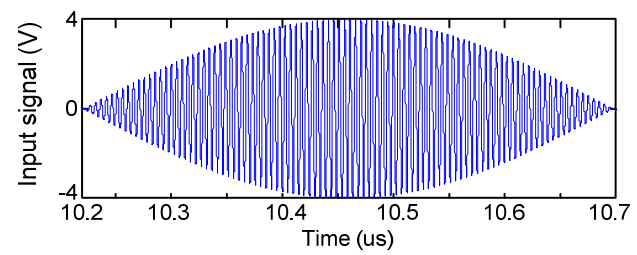

(a)

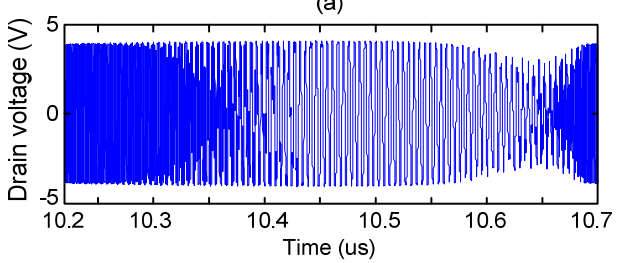

(b)

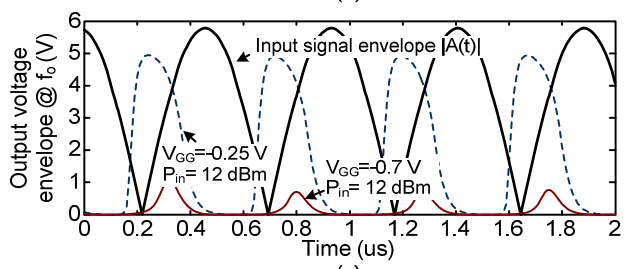

(c)

Fig. 10 Influence of the input signal on the self-oscillation. (a) Input signal: two tones with $\Delta f \approx 2 \mathrm{MHz}$. (b) Time-domain analysis of the SOPA with $V_{G G}=-0.7 \mathrm{~V}$. (c) Envelope analysis. Amplitude at the oscillation frequency. 


\section{CONCLUSION}

A brief description of iterative nonlinear simulation methods has been presented, with emphasis on oscillation and instability problems. The methods have been illustrated with timely circuit examples of complex behavior, such as pulse injection-locked oscillators, rotary travelling wave oscillators and unstable power amplifiers.

\section{ACKNOWLEDGEMENT}

Author is grateful to Franco Ramírez, Sergio Sancho and Mabel Pontón from University of Cantabria. This work has been supported by the Spanish Government under contract TEC2014-60283-C3-1-R and the Parliament of Cantabria (12.JP02.64069).

\section{REFERENCES}

[1] J. M. T. Thompson, H. B. Stewart, Nonlinear dynamics and chaos, John Wiley \& Sons, 1986.

[2] J. Guckenheimer, P. Holmes, Nonlinear oscillations, dynamical systems and bifurcations of vector fields, Springer-Verlag, 1990.

[3] J. C. Pedro, N. Carvalho, Intermodulation distortion in microwave and wireless circuits, Artech House Inc, 2002.

[4] M. Schetzen, The Volterra \& Wiener Theories of Nonlinear Systems, John Wiley \& Sons, New York, 1980.

[5] D. O. Pederson, "A historical review of circuit simulation," IEEE trans. on Circuits and Systems, vol. 31, no.1, Jan. 1984.

[6] K. S. Kundert, "Introduction to RF simulation and its application," IEEE Journal of Solid State Circuits, vol. 34, no. 9, pp. 1298-1319, Sep. 1999.

[7] K. S. Kundert, A. Sangiovanni-Vicentelli, "Finding the steadystate response of analog and microwave circuits," IEEE Custom Integrated Circuits Conference, 1998.

[8] M. I. Shoby, A. K. Jastrzebsky, "Direct integration methods of nonlinear microwave circuits," 15th European Microwave Conference Proceeding, Paris 1985, pp. 1110-1118.

[9] C. Camacho-Peñalosa, "Numerical steady-state analysis of nonlinear microwave circuits with periodic excitation," IEEE Trans. Microw. Theory and Techn., vol. 31, no. 9, pp. 724-730, Sep. 1983.

[11] V. Rizzoli, A. Neri, "State of the art and present trends in nonlinear microwave CAD techniques," IEEE Trans. Microw. Theory and Techn, vol. 36, no. 2, Feb. 1988, pp. 343-356.

[12] R. Quéré, E. Ngoya, M. Camiade, A. Suarez, M. Hessane, J. Obregon, "Large signal design of broadband monolithic microwave frequency dividers and phase-locked oscillators," IEEE Trans. Microw. Theory and Techn. vol. 41, no. 11, pp. 1928-1938, Nov. 1993.

[13] T. S. Parker, L. O. Chua, Practical algorithms for chaotic systems, Springer-Verlag, Berlin, 1989.

[14] J. Bonet, P. Palá, J. M. Miró, “A discrete-time approach to the steady state analysis of distributed nonlinear autonomous circuits," IEEE Int. Symp. on Circuits and Systems, pp. 460-464, pp. 1998.

[15] I. Maio, F. G. Canavero, "Differential-difference equations for the transient simulation of lossy MTLs," IEEE Int. Symp. on Circuits and Systems, vol. 2, pp.1402-1415, 1995.

[16] A. Dounavis, L. Xin, M. S. Nakhla, R. Achar, "Passive closedform transmission-line model for general-purpose circuit simulators," Microwave Theory and Techniques, IEEE Transactions on, vol. 47, no. 12, pp. 2450,2459, Dec 1999.
[17] T. Brazil, "Nonlinear, "Transient Simulation of Distributed RF Circuits using Discrete-Time Convolution," IEEE Int. Symp. on Circuits and Systems, pp.505,508, 27-30 May 2007

[18] A. Suárez, R. Quéré, Stability Analysis of Nonlinear Microwave Circuits. Artech House, Norwood (Ma), Jan. 2003.

[19] A. Suarez Analysis and design of autonomous microwave circuits, Wiley-IEEE Press, 2009.

[20] J. Jugo, J. Portilla, A. Anakabe, A. Suárez, and J. M. Collantes, "Closed-loop stability analysis of microwave amplifiers," IEE Electronics Letters, vol. 37, pp. 226-228, Feb. 2001.

[21] A. Anakabe, J. M. Collantes, J. Portilla, et al. "Analysis and elimination of parametric oscillations in monolithic power amplifiers," IEEE Int. Microwave Symp. Seattle, Jun. 2002, pp. 2181-2184.

[22] N. Deparis, A. Siligaris, P. Vincent, N. Rolland, "A 2 pJ/bit pulsed ILO UWB transmitter at $60 \mathrm{GHz}$ in 65-nm CMOS-SOI," IEEE International Conference on Ultrawideband, Oct. 2009, pp. 113-117.

[23] E. Fernández, F. Ramírez, A. Suárez, S. Sancho, "Stability and phase-noise analysis of pulsed injection-locked oscillators," IEEE Trans. Microw. Theory Techn., vol. 61, no. 1, pp. 482491, Jan., 2013.

[24] J. Wood, T. C. Edwards, S. Lipa, "Rotary traveling-wave oscillator arrays: A new clock technology," IEEE J. Solid-State Circuits, vol. 36, no. 11, pp. 1654-1665, Nov. 2001.

[25] M. Pontón, A. Suárez, J.S. Kenney, "Analysis and design of Rotary Traveling Wave Oscillators using Nonlinear Transmission Lines," IEEE Trans. Microw. Theory Techn., vol. 62, no. 5, pp. 1149 - 1161, May. 2014.

[26] C. W. Ho, A. E. Ruelhi, P. A. Brennan, "The modified nodal approach to network analysis," IEEE trans. on Circuits and Systems, vol. 22, no.6, Jun 1975.

[27] H. Dag, F. L. Alvarado, "Computation-free preconditioners for the parallel solution of power system problems," IEEE Trans. on Circuits and Systems, vol. 12, no. 2, May 1997. pp. 585-591.

[28] R. W. Freund, "Passive reduced-order modelling via Krylovsubspace methods," IEEE International symposium on Computer-Aided Control System Design, Anchorage, Alaska, Sep. 2000, pp. 261-266.

[29] R. Melville, H. G. Brachtendorf, "An effective procedure for multi-tone steady-state analysis of mixers," the 8th IEEE Conference on Electronics Circuits and Systems, vol. 3, pp. 1449-1253, 2001.

[30] E. Ngoya, R. Larcheveque, "Envelope transient analysis: Anew method for the transient and steady state analysis of microwave communication circuits and systems," Proc. of IEEE MTT Symposium, San Francisco, Jun. 1996.

[31] H. G. Brachtendorf, G. Welsch, R. Laur, "A novel timefrequency algorithm for the simulation of the steady state of circuits driven by multi-tone signals," IEEE International Symp. on Circuits and Systems, Hong-Kong, Jun. 1997, pp. 1508-1511.

[32] F. Ramírez, A. Suárez, I. Lizarraga, J. M. Collantes, "Stability analysis of nonlinear circuits driven with modulated signals," IEEE Trans. Microw. Theory Techn., vol. 58, no. 4, pp. 929940, Apr. 2010.

[33] M. Sarkeshi, R. Mahmoudi, and A. van Roermund, "Efficiency analysis of a limit-cycle class-D amplifier with a random Gaussian excitation," IEEE Int. Micro. Symp. Dig., pp. 13691372,2009

[34] A. Suárez, E. Fernández, F. Ramírez, S. Sancho, "Stability and bifurcation analysis of self-oscillating quasi-periodic regimes," IEEE Trans. Microw. Theory Techn., vol. 60, no. 3, pp. 528541, Mar., 2012. 